\title{
Quaderni
}

QUADERNI Communication, technologies, pouvoir

79 | Automne 2012

Produire la démocratie

\section{La métropole parisienne mise en images sur Internet : regards publics et prismes médiatiques}

Dominique Pagès

\section{CpenEdition}

\section{Édition électronique}

URL : http://journals.openedition.org/quaderni/632

DOI : 10.4000/quaderni.632

ISSN : 2105-2956

\section{Éditeur}

Les éditions de la Maison des sciences de l'Homme

\section{Édition imprimée}

Date de publication : 5 octobre 2012

Pagination : 119-128

\section{Référence électronique}

Dominique Pagès, «La métropole parisienne mise en images sur Internet : regards publics et prismes médiatiques », Quaderni [En ligne], 79 | Automne 2012, mis en ligne le 05 octobre 2014, consulté le 02 mai 2019. URL : http://journals.openedition.org/quaderni/632 ; DOI : 10.4000/quaderni.632 


\section{Communication}

\section{La métropole parisienne \\ mise en images sur Internet : regards publics et prismes médiatiques}

Le processus de métropolisation parisien s'appuie sur une diversité d'acteurs institutionnels et médiatiques dont la plupart sont récents voire émergents mais qui proposent tous soit de simples contenus en ligne, soit un site internet pour exposer leur vision, leur point de vue, leurs travaux. Autant de voix et de regards qui commentent le projet, ses dispositifs et ses méthodes et participent à sa publicisation.

Envisager cet espace public numérique en continu - et notamment les écritures visuelles qui participent à l'énonciation de la métropole en devenir -, c'est non seulement répondre à un souci de visibilité et de lisibilité mais aussi de questionnement des imaginaires en présence : quelles images sont privilégiées sur Internet pour signifier le projet de métropolisation, pour évoquer un « territoire en émergence »? Quel regard sur l'habitant et l'habiter métropolitain proposent-ils ? Dans quelle mesure ces images (ou leur absence) révèlent-elles la complexité et la difficulté d'une représentation métropolitaine?

Un «système» communicationnel en constitution

\section{Dominique Pagès}

Maître de Conférences CELSA, GRIPIC Université Paris Sorbonne
Dans un premier temps envisageons les contenus iconographiques proposés par les acteurs qui énoncent et discutent la métropolisation, celle de Paris mais aussi le processus en général.

\section{La DPMC ${ }^{1}$ : une communication de projet qui cherche à s'émanciper?}

La délégation générale à Paris métropole et aux coopérations interterritoriales (DPMC), actrice historique ayant progressivement installé la 
question métropolitaine dans l'agenda politique, est chargée de mettre en œuvre la démarche d'ouverture et de coopération avec les collectivités voisines. Elle fonde sa démarche sur trois objectifs stratégiques : organiser la métropole au quotidien en développant des solidarités territoriales en zone dense ; participer au processus et affirmer Paris comme un des acteurs majeurs de Paris métropole ; favoriser l'émergence d'une culture métropolitaine grâce à des évènements partagés. Elle est organisée en trois pôles : le «pôle Coopérations », le «pôle Métropole» et le « pôle Pratiques, cultures, projets et évènements partagés ».

L'analyse de l'évolution des choix communicationnels et plus spécifiquement iconographiques de la délégation demanderait un long et scrupuleux travail de thèse, tant les signes et les actions se sont multipliés, ajustés, concrétisés avec le temps. Mais, globalement, il semble que la communication de la délégation qui a donné le rythme à l'agenda métropolitain pendant dix années reste celle d'un projet territorial de grande envergure, accompagné par des expertises croissantes.

La délégation n'a pas de site propre (elle a transféré la plupart de ses fonds documentaires vers le site du syndicat Paris Métropole) mais a une entrée sur le site de la Mairie. Son action est surtout relayée par des acteurs partenaires : le syndicat Paris Métropole donc mais aussi l'APUR (l'agence parisienne d'urbanisme, www. apur.org) ${ }^{2}$ dont le rôle est déterminant. Grande productrice de cartes, l'Agence d'urbanisme de Paris traduit ainsi la démarche métropolitaine et organise régulièrement avec le Pavillon de l'Arsenal ${ }^{3}$ des expositions intégrant divers sup- ports iconographiques : maquettes, films, catalogues. Les logiques urbanistique et géographique restent donc prépondérantes dans les choix iconographiques. Mais la DPMC fait appel dans ses publications papiers (d'Extra Muros aux Cahiers de la Métropole) au regard des artistes (photographes, documentaristes, écrivains) pour donner une vision plus tangible de la métropole vécue.

\section{Le syndicat Paris Métropole (www.parismetro- pole.fr) : une jeune institution communicante}

Créé en 2009, ce Syndicat mixte d'études, rassemblant les collectivités membres et associant le plus légitimement possible les acteurs du territoire en devenir, fut d'abord une émanation de la Délégation, inscrit dans le sillage de la Conférence Métropolitaine. Présidé par un maire de la périphérie de Paris (alternance gauche/droite), ses travaux sont à dimension tout à la fois stratégique et opérationnelle et s'organisent autour de quatre axes prioritaires : développement et solidarité, déplacements, logements, projets métropolitains.

Devant œuvrer à la création durable d'une communauté d'élus (193 collectivités adhérentes à l'automne 2011) mais aussi d'un réseau généraliste autour de la métropolisation, ses choix communicationnels ont évolué. Le syndicat dit vouloir faire grandir « la conscience métropolitaine »; pour cela il lui faut signifier la communauté, l'aider à construire et à partager des connaissances ainsi qu'une culture communes. Le choix d'un logo traduisant, par un jeu d'entrelacements, la logique d'interterriorialité et, par ses couleurs enjouées (rose, vert), un souhait de sortir des codes de la communication publique, puis le lancement d'un site internet, modeste mais constituant progres- 
sivement un centre ressources incontournable (à la fois grand public mais préservant des espaces protégés pour les membres) ont d'emblée marqué une nouvelle manière d'écrire un projet de territoire, tant dans le ton, le style que dans certains contenus (remontées d'expériences, de paroles d'élus et d'experts inaccoutumés).

Par ailleurs, le syndicat qui se veut le creuset des échanges sur la thématique de la gouvernance métropolitaine a lancé dès 2010 un « appel à initiatives » ouvert à l'ensemble des membres : celuici a permis aux acteurs locaux de faire apparaître via un site dédié ${ }^{4}$ des projets locaux parfois très imagés et localisés, contrastant avec les images du site du Syndicat, relativement abstraites (présence des cartes : celle des membres, celle du schéma d'ensemble du Grand Paris, celle des Contrats de développement territorial prévus par la loi Grand Paris du 3 juin 2010), et « attendues » (ainsi les images présentant les commissions : quai de gare TGV, assemblée métropolitaine, tours, la Défense, tarmac d'aéroport).

\section{La Société du Grand Paris (www.societedu- grandparis.fr) : une austère rhétorique de la vulgarisation?}

Cet établissement public a été créé à l'initiative de l'État en juin 2010 (après la création d'un secrétariat d'État au développement de la « Région Capitale »). Chargé de concevoir le métro du Grand Paris en 2013, reliant les banlieues entre elles, de mettre en œuvre le réseau de transports du Grand Paris, cet établissement a organisé les débats publics relatifs aux projets Arc Express et réseau du Grand Paris (en septembre 2010) sous l'égide de la Commission Nationale du Débat public (CNDP).
Son site est encore assez pauvre en termes d'iconographie métropolitaine : les images sont convenues (grand ensemble vu du ciel, groupe d'élus non identifiés, tracés et cartes, schémas, atlas cartographique, rame de métro vide, escalators où se devinent les flux tremblés de passagers, jambes de passants pour signifier la marche, silhouette isolée regardant derrière une vitre les avions d'un aéroport anonyme, simulation d'une future gare). Ce choix semble plus alimenté par une banque d'images que par une production spécifique aux territoires de la métropole parisienne.

\section{L'AIGP (www.ateliergrandparis.com) : une stratégie visuelle offensive mais encore désor- donnée}

Créé en 2010 dans le sillage de la consultation de 2008 et installé au Palais de Chaillot, l'atelier international du Grand Paris (un GIP, groupement d'intérêt public qui associe à parité État et Collectivités territoriales et dont le Conseil scientifique est composé des dix équipes qui ont travaillé depuis 2008 sur le projet) a pour vocation de devenir un centre de partage et de promotion des recherches en aménagement, urbanisme et architecture durables autour du Grand Paris. Son objectif est aussi de stimuler la création architecturale et urbaine en Europe en lançant des appels à idées ouverts aux jeunes concepteurs européens, en animant réflexions et débats sur les nouvelles pratiques de projets avec tous les acteurs de la ville, en multipliant les études thématiques ${ }^{5}$, en soutenant et évaluant les réalisations exemplaires. L'AIGP qui est organisé autour de quatre ateliers regroupant architectes, urbanistes, économistes, artistes ('nouvelle géographie de Paris', 'Habiter plus \& habiter mieux', 'Grand Paris écologique', 
'aménager la Seine') a manifesté dès sa création la volonté de « libérer la création ».

Son site internet, en refonte à l'automne 2011, privilégie l'image, les recherches iconographiques sur diverses problématiques du Grand Paris : en partenariat avec les écoles d'architecture mais aussi avec l'IGN, l'AIGP veut favoriser le déploiement d'un site géoportail spécifique au Grand Paris, permettant notamment de géolocaliser les territoires de projet et d'approfondir le travail cartographique, comme outil de vulgarisation du fait métropolitain. De plus, l'Atelier énonce sa volonté de mener, avec l'assistance de l'APUR et de l'IAURIF (l'Institut d'aménagement et d'urbanisme d'Île de France, www.iaurif.org/www. iau-idf.fr), un travail spécifique de représentation du paysage urbain hérité dans l'agglomération parisienne : l'objectif est de signifier la diversité des tissus urbains, les grandes familles de territoires (villes historiques sédimentées, zones pavillonnaires, tissus industriels, territoires des grands ensembles, centres villes néo-faubouriens, périphéries, etc.) dans leur inscription historique et géographique. Un travail qui pourrait déboucher sur la constitution d'un Atlas métropolitain, le premier inventaire de la métropole.

La communication naissante de l'AIGP a donc recours aux images et semble en quête d'une vision d'ensemble, d'une unité de sens à restituer au grand public. Nous retrouvons ici un écho aux discours utopiques du XIX ${ }^{\mathrm{e}}$ siècle où l'architecture était pensée comme apte à changer les rapports sociaux et où les visions de villes nouvelles et réformistes se multiplièrent (de Saint-Simon à Charles Fourier, de Nicolas Ledoux à Victor Considérant, autant de projets utopistes dont les images hantent encore notre imaginaire commun).

L'atelier qui semble vouloir innover d'un point de vue technologique et esthétique dans la représentation des projets (3D, vidéos, simulations, cartographie dynamique, etc.) met donc au cœur de ses missions la «culture de l'image» : représenter la métropole à travers les lieux emblématiques à valoriser ou à créer.

\section{Le Conseil régional et l'IAU (www.iaurif.org /www.iau-idf.fr) : un vivier d'images expertes et ordinaires}

Le conseil Régional est depuis longtemps une partie prenante de la métropolisation qui, à la fois, l'accompagne et se protège d'une possible mise à mal de ses limites et prérogatives. En termes iconographiques, l'IAURIF est central puisqu'il produit, édite cartes, schémas, photographies, à la fois actuelles et prospectives, qu'il diffuse sur son site mais aussi via nombre de publications : ainsi les Cahiers dont quatre numéros mettent au centre la question métropolitaine à force d'images : les numéros 160 ('Gouvernance(s) et ingénierie métropolitaines'), 157 ('Equipements et services : la métropole au quotidien'), 151 ('Stratégies métropolitaines'), 127 et 128 ('Les transports dans les grandes métropoles'), et plus particulièrement le numéro 158 de ses Cahiers ('Et demain?') qui se propose de réfléchir à la vision métropolitaine à 50 ans. En demandant à des spécialistes, des artistes, des experts d'imaginer ce que sera la ville de demain, ce numéro questionne et actualise les imageries en place.

\section{L'AMIF (www.amif.asso.fr) : l'arlésienne métropolitaine}


L'association des maires d'Île de France qui regroupe l'ensemble des élus de la grande et de la petite couronne a un rôle de représentation des élus locaux et donc de porte-parole. C'est un interlocuteur privilégié sur les grandes questions qui conditionnent l'avenir de la région voire $\mathrm{du}$ pays. Mais si l'association donne son avis sur le développement de la région Capitale, sur les projets de réseau de transport public du Grand Paris et sur l'ensemble des autres projets, son site ne témoigne guère d'une prise en compte iconographique de ceux-ci.

Une rapide synthèse des choix de communication des différents énonciateurs publics, en termes d'iconographie territoriale, met en évidence quelques grandes tendances :

- l'importance des logos signifiant les différentes visions en présence d'un territoire en devenir : pour Paris métropole, l'interritorialité ; pour le projet Grand Paris (qui a récemment dévoilé son troisième logo, repenti des deux premiers), tour à tour, la polycentralité (trois cercles concentriques plus ou moins larges se juxtaposant pour signifier l'agencement de centres plus ou moins influents) puis la mosaïque de micro-territoires pour dire la multitude des engagements et enfin le logo de marque émergent (jouant sur le symbole de Tour Eiffel dédoublé).

- la forte présence de la carte «dans tous ses états» et des schémas : depuis son origine, le projet de métropolisation s'est traduit par des cartes, de celles produites dès la fin du XIX ${ }^{\mathrm{e}}$ siècle juste après l'annexion de 1860 (le passage de 11 à 20 arrondissements) à celles diffusées aujourd'hui à profusion. Les cartes, qu'elles soient en pétales de marguerite, en étoile, rhyzomiques, fixes ou dynamiques, sont omniprésentes dans la communication métropolitaine, sans doute parce qu'elles rassurent par leur rationalité et esthétisent le processus, leurs couleurs le rendant ludique, les ondulations en disant le mouvement, les symboles (flèches, liens...) évoquant les métamorphoses d'un territoire. Les cartes disent bien la diversité des regards disciplinaires (cartes des géographes, des architectes, des urbanistes, des sociologues) et donc des compétences professionnelles convoquées (cartographes, infographistes, communicants, mais aussi artistes). Ces cartes souvent spectaculaires ne constituent donc pas véritablement des signes d'unité pour les habitants. De plus, elles donnent à penser avec les schémas un programme planifié alors que le projet de Métropole ne relève pas véritablement d'une planification ou d'une programmation mais constitue bien une approche stratégique qui, certes, établit des scénarios d'action mais modifie son action en fonction des informations recueillies, des échanges et des débats. Ce qui ne fait pas le programme : la carte rassure plus qu'elle ne restitue la complexité de la vision politique ou stratégique de la métropolisation.

- la place des images issues de la consultation des architectes : celle-ci marqua un tournant incontestable et un changement d'échelle de la communication. Jusqu'ici confidentielle et discrète, la question de la métropolisation devint alors sociétale et «civilisationnelle», de portée internationale dont seule une mise en spectacle épique pouvait traduire l'ambition et la portée. Sur la plupart des sites publics se retrouvent encore certains des visuels produits par les dix équipes qui circulèrent dans les médias tant 
nationaux qu'internationaux et marquèrent en profondeur les imaginaires de chacun. Mais, progressivement, ils laissent la place à d'autres, clairement réalistes ou bien poétiques, reliés à des territoires connus.

- la présence inégale des portraits d'acteurs et des habitants : la représentation concrète des habitants fut longtemps absente ou implicite dans la communication métropolitaine, bien que la délégation se soit très tôt efforcée de signifier l'importance de sa place et sa diversité au fil des publications. L'évocation du quotidien, de ses scènes et de ses rites reste souvent un enjeu de mots plus que d'images. Mais, sans doute, l'habiter métropolitain reste encore pour bon nombre plus «subi» que «voulu», très inégalitaire dans ses formes, ses espaces et ses temporalités : les deux à trois heures de transports journaliers pour les « banlieusards», le coût du budget automobile pour les périurbains, le manque de mètres carrés pour les familles parisiennes sont des réalités qu'aucune image publique enchantée célébrant les aménités métropolitaines ne saurait résorber. De là, la prédilection pour les images du futur sur celles de l'ordinaire des individus, les mots étant là pour dire plus la situation actuelle et sa complexité. Si les mots énoncent plus clairement le diagnostic, les images suggèrent le dépassement des référents en place, l'interdépendance des territoires, leur connexité et leur contiguité.

\section{Les choix visuels d'autres communicants, experts et médiatiques}

Pour compléter cette présentation des sites des principaux acteurs publics de la métropolisation parisienne, il est éclairant de nous tourner vers d'autres promoteurs et commentateurs de ce processus et des politiques qui le portent. Parmi eux, les acteurs publics dits fonctionnels (RFF, $\mathrm{CAUE}^{6}$, etc.) mais aussi les marchands (RATP, Decaux, Véolia et son observatoire des modes de vie urbain, etc.). Nous ne retiendrons ici que deux catégories dont le positionnement et donc les choix éditoriaux sont nécessairement différents, tant en termes textuels que visuels : les sites et revues d'experts qui analysent, théorisent, mettent en débat la métropolisation, veulent comparer le cas parisien et plus largement français à d'autres expériences de métropolisation (européennes ou mondiales) ; les médias marchands qui innovent dans les choix iconographiques.

\section{Métropoles revue (http ://metropoles.revues. org) : l'absence déplorée}

Cette revue en ligne dont le rédacteur en chef est le chercheur Christian Lefebvre, co-responsable du Latts ${ }^{7}$, se veut le carrefour des travaux scientifiques issus de la plupart des disciplines en sciences sociales qui s'intéressent à la métropolisation, et avant tout aux questions de gouvernance. Elle donne place aux articles et aux produits scientifiques originaux ayant une dimension internationale et comparative forte. Si elle vise à être le lieu d'un débat sur l'avenir des sociétés urbaines, elle n'offre aucune vision imagée de la métropolisation, du projet et du processus.

\section{Métropolitiques (www.metropolitiques.eu) : les vignettes, un territoire en éclats}

Cette revue électronique créée en 2010 (et complétée par une newsletter), à l'initiative de Frédéric Gilli, économiste et géographe, directeur 
délégué de la chaire Ville à Sciences Po, s'est tout d'abord proposé de questionner le pilotage politique du projet parisien mais multiplie depuis les points de vue sur l'actualité métropolitaine dans le monde, qu'elle soit politique, culturelle ou urbanistique. Analysant l'insertion des grandes métropoles et les évolutions de leur organisation interne, la revue cherche à la fois à confronter «les savoirs et les savoir faire sur la ville» et sur «l'architecture et les territoires» mais aussi à faire remonter les expériences de terrain liées au monde professionnel, politique ou associatif. Animée par des enseignants chercheurs et des praticiens issus de la plupart des disciplines de l'urbain et d'ambition internationale (appui d'un réseau de correspondants en France et à l'étranger), elle met en ligne débats, essais, commentaires, entretiens, billets d'humeur.

Ses choix iconographiques ont évolué en une année : le logo reste très marqué par l'idée de la diversité du bâti et de l'imbrication des formes d'habitat. Mais les rares vignettes du début se sont enrichies sans chercher pour autant une ligne éditoriale claire : portraits d'interviewés, couvertures des ouvrages et des études cités, bris de carte, fragments urbains, éclats d'espaces publics non localisés, etc. se distillent pour illustrer des titres qui, à eux seuls, «font image» ('Sentier d'été', 'Summer break', 'City words : an invitation to travel', 'Southland ou la traversée des frontières de Los Angeles, 'manger local, une utopie concrète', etc.).

\section{Les Métropolitaines (www.lesmetropolitaines. eu) : le logo comme manifeste}

Ce site minimaliste est celui d'un groupe de réflexion et de lobbying sur la métropolisation, lancé au début de l'année 2011 à l'initiative de Vincent Feltesse (président de la Communauté Urbaine de Bordeaux) et d'Olivier Mongin (directeur de la revue Esprit, qui veut contribuer depuis longtemps à la promotion de la culture urbaine en France). Le groupe veut « mettre à l'agenda politique, médiatique et scientifique la question de la métropolisation », " interpréter pour sensibiliser et faire émerger un débat public », encourager la création d'actions et d'évènements métropolitains. Le seul visuel est le logo très géométrique, signifiant la mise en réseau de points métropolitains (approche polynucléaire mettant à égalité chacune des villes métropolitaines) que ceux-ci soient franciliens, français ou européens.

\section{Échos de Métropole (http ://echosdemetropole. caue69.fr) : le talent des dessinateurs}

Si les réflexions proposées sur ce blog par un chercheur (missionné au CAUE local) se focalisent surtout sur la métropole lyonnaise, elles envisagent aussi la métropolisation parisienne ainsi que celle des villes européennes. Le blog multiplie de plus les liens vers des sites spécialisés sur les transformations urbaines (et notamment : 'branding my city', 'branding the city', 'le forum lyonnais des grands projets urbains', 'urbablog', 'urbains sensibles'). Par ailleurs, il propose à des illustrateurs (Jérémy Gravayat, Adrien Cacheux, Pierre Ferrero, Rudy Guedj) de traduire par le dessin leur vision de la métropole mais aussi à de jeunes documentaristes de poster leur courts et moyens métrages sur la métropole. La variété des interprétations visuelles et des genres enrichit ce site oscillant entre centre ressources, site expert et plateforme plurimédias. 
Si la rhétorique de ces sites n'invite guère à une lecture iconographique construite du processus (par choix ou par manque de moyens), deux titres de médias plus grand public, de création relativement récente, proposent des approches visuelles plus fournies, assumées et contrastées.

\section{La revue Place Publique (www.revue.placepu- blique.fr) : la Métropole vivante}

Cette « revue urbaine » de débat et de réflexion sur les questions urbaines et métropolitaines née à Nantes Saint-Nazaire à l'initiative de Thierry Guidet (et existant depuis peu à Rennes) s'est rapprochée en 2010 de la DPMC pour lui proposer une déclinaison parisienne. Elle se positionne comme un média local devant accompagner le déploiement d'un territoire complexe. Au cœur de ce positionnement, la volonté de «privilégier la raison sur l'émotion», «la durée et non l'instant», «la confrontation des projets», «en croisant savoirs, regards et approches».

Cette revue (marchande mais répondant à une commande publique) affiche des choix thématiques et visuels très concrets reliés au territoire de référence, d'aujourd'hui, d'hier et de demain. Si le comité de rédaction est composé de nombre d'universitaires, les contenus sont fortement éditorialisés, les couvertures illustrant le style très journalistique de la revue : elles donnent à voir dans un bandeau central des hommes, des lieux, des situations (politiques, festives, quotidiennes, d'hier ou d'aujourd'hui), des fragments emblématiques de territoires et parfois, des visuels signifiant l'avenir.

Le magazine " Mégalopolis", magazine du très
Grand Paris (www.megalopolismag.com) : la métropole des jeunes

Ce magazine trimestriel d'enquête, de reportages et de récits sur l'Île-de-France et le Grand Paris, a été créé en 2010 à l'initiative d'étudiants en sciences politiques. Partant du constat de l'absence d'une offre médiatique " grand public» sur la métropole en devenir, il veut devenir «le magazine de référence de la région parisienne, celui qui donne le sens au Grand Paris ».

Jouant sur la complémentarité papier/internet, il propose des reportages au ton décalé, des analyses décomplexées et très accessibles de la métropole, de ses situations, de ses lieux, de ses acteurs. Ses couvertures souvent insolites créent une rupture avec le traitement médiatique de la métropolisation par les médias publics et les magazines généralistes : un pied géant en basket franchissant le périphérique, un individu déguisé en panda dans une rue pavillonnaire, une jeune femme en tenue de plage, pieds nus et équipée d'une grande bouée, attendant sur un quai RER... autant d'images qui disent le souci d'incarner la métropole par des figures décalées de jeunes habitants jouant avec le territoire.

De même, le site internet d'actualité mélange les genres et les visuels (cartes, portraits, scènes de vie quotidienne, etc.). Une application payante Mégalopolis est disponible sur Iphone, agrégeant les contenus du site web et du magazine. On peut naviguer dans la liste d'articles via leurs photographies : un diaporama se lance et on retrouve l'article en cliquant sur la photo.

Ces sites, revues et magazines, offrent donc des 
approches contrastées de l'écriture visuelle des territoires métropolitains oscillant entre volonté de rendre compte du processus et souci de témoigner des vécus métropolitains : si les sites experts mettent plutôt au centre les commentaires et analyses (certains s'ouvrant discrètement à un travail d'éditorialisation plus complet), la plupart sortent des images chimériques issues de la consultation pour offrir au fil de fragments métropolitains des prises sur un territoire tout à la fois local et mondial.

Cette présentation des principaux énonciateurs métropolitains constitue une invitation à penser plus avant les choix visuels, oscillant entre absence énigmatique et saturation désordonnée, imaginaire spectaculaire et imaginaire ordinaire, à comprendre plus finement le statut de l'image numérique dans l'écriture du processus, de sa création à sa diffusion et circulation, les effets de sens qu'elle suscite, dans un souci de pédagogie du processus ${ }^{8}$.

\section{$R \cdot E \cdot F \cdot E \cdot R \cdot E \cdot N \cdot C \cdot E \cdot S$}

BOYER H. et CARDY H. (sous la direction de), "Les collectivités territoriales en quête d'identité », revue Mots-les langages du politique, n87, novembre 2011.

MAIRIE DE PARIS, Les cahiers de la métropole, $\mathrm{n}^{\circ} 1$.

PAGÈS, D. (sous la direction de), « La Métropole parisienne : entre récits, paroles et échanges », Quaderni, nº73, automne 2010. 


\section{$\mathrm{N} \cdot \mathrm{O} \cdot \mathrm{T} \cdot \mathrm{E} \cdot \mathrm{S}$}

1. Site de la mairie de Paris, mais aussi le blog de l'élu, un centre ressources : www.pierremansat.com 2. www.apur.fr : association créée en 1967, subventionnée par la mairie parisienne qui a pour vocation de fournir des études pointues sur l'aménagement du territoire francilien.

3. www.pavillon-arsenal.com

4. www.parismetropole.fr/nos-chantiers/initiativesmetropolitaines/appel-a-initiatives.

5. Les thèmes actuellement privilégiés sont : les équilibres métropolitains, les fractures de la métropole (qui contraignent et freinent le développement urbain et social des territoires), la densité et les formes urbaines, la nature et les paysages métropolitains.

6. Le conseil d'architecture, d'urbanisme et de l'environnement de Paris (www.caue75.fr) mais aussi l'Union Régionale des CAUE (www.urcaue. fr) offrent des formations et des voyages d'études dédiés à la métropolisation, permettant la diffusion et les échanges de savoirs, le décryptage des enjeux et l'acquisition pour les stagiaires - élus et fonctionnaires - des outils techniques et pratiques de mise en œuvre des politiques d'aménagement.

7. Chercheur au LATTS mais aussi directeur de l'Institut Français d'Urbanisme (IFU) et professeur à l'Université de Paris Est Marne La Vallée.

8. http ://imagesdesterritoires.univ-pau.fr/live/ 